\title{
Un siècle de publicité horlogère, 1880-1980
}

L'exposition L'heure pour tous, une montre pour chacun au Musée international d'horlogerie, La Chaux-de-Fonds, Suisse

\section{Catherine Cardinal}

\section{Q OpenEdition \\ 12 Journals}

\section{Édition électronique}

URL : http://journals.openedition.org/artefact/4787

DOI : $10.4000 /$ artefact. 4787

ISSN : 2606-9245

Éditeur :

Association Artefact. Techniques histoire et sciences humaines, Presses universitaires du Midi

\section{Édition imprimée}

Date de publication : 15 décembre 2019

Pagination : 193-196

ISBN : 978-2-8107-0667-9

ISSN : 2273-0753

\section{Référence électronique}

Catherine Cardinal, «Un siècle de publicité horlogère, 1880-1980 », Artefact [En ligne], 11 | 2019, mis en ligne le 27 novembre 2020, consulté le 29 novembre 2020. URL : http://journals.openedition.org/ artefact/4787 ; DOI : https://doi.org/10.4000/artefact.4787

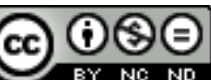

Artefact, Techniques, histoire et sciences humaines est mise à disposition selon les termes de la Licence Creative Commons Attribution - Pas d'Utilisation Commerciale - Pas de Modification 4.0 International. 


\section{Un siècle de publicité horlogère, $1880-1980$}

\section{L'exposition L'heure pour tous, une montre pour chacun au Musée international d'horlogerie, La Chaux-de- Fonds, Suisse}

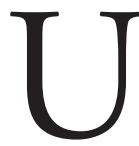

Tn univers visuel, habituel à nos yeux à travers l'affichage urbain et la publicité des magazines, mais encore inexploré en tant que domaine de recherches, a été mis en valeur dans le cadre du Musée international d'horlogerie : celui des affiches et des annonces produites par les industriels de l'horlogerie. Les limites chronologiques de la production ont été choisies selon deux faits marquants de l'évolution de la montre : le développement de la montre-bracelet à la fin du XIX ${ }^{e}$ siècle et l'invention de la montre à quartz, un siècle plus tard.

Entre ces deux périodes, la communication des maisons horlogères prend un essor considérable dont témoignent d'innombrables images publicitaires créées par chacune d'elles pour se démarquer de ses concurrentes. La fantaisie, l'humour, la loquacité, l'austérité, l'effet de surprise, tous les moyens sont bons pour attirer le regard des clients potentiels. La séduction des couleurs, la sûreté du graphisme et l'insertion judicieuse de formules laconiques ou de petits textes contribuent à la réussite des messages. Sans entrer dans un discours historique, le visiteur de l'exposition peut assurément être ravi de découvrir les cent façons de mettre en scène un produit qui lui est familier ${ }^{1}$. Mais, l'objectif de la manifestation n'est pas seulement d'assouvir la curiosité et de susciter un plaisir esthétique. Son but est aussi de présenter les fruits d'une recherche, commencée en 2015,

1. Exposition présentée du 16 juin au 13 octobre 2019.

95 Catherine Cardinal, « Un siècle de publicité horlogère, 1880-1980 », Artefact, 11, 2019, p. 193-196. 
grâce à un partenariat entre le Musée international d'horlogerie et l'Institut d'histoire de l'Université de Neuchâtel, ayant abouti à la collecte d'environ 2500 documents publicitaires dont $10 \%$ ont été sélectionnés pour être présentés ${ }^{2}$.

L'apparent foisonnement des grands panneaux publicitaires mis en place par le scénographe est structuré selon un plan rigoureux, révélant l'histoire culturelle de l'affiche horlogère des années 1880 aux années 1980. Six thématiques ressortent dans la création des images et des textes qui leur sont indissociables ${ }^{3}$. La première section s'attache à la représentation de la notion du temps. Le cours du jour, les astres, le zodiaque, les saisons inspirent les affichistes pour donner un environnement grandiose à la montre (Fig. III, cahier couleur) ; le personnage allégorique du Vieillard-Temps donne un ton dramatique à des compositions influencées par le symbolisme des années $1880^{4}$. La deuxième section rappelle que l'exactitude et la fiabilité sont indispensables dans un monde désormais régi par la vitesse et révolutionné par les transports. Au milieu du siècle, l'image de l'avion est souvent efficacement associée à celle de la montre de précision. Dans une troisième partie, la référence aux innovations qui scandent le perfectionnement des montres s'impose dans la réclame de toutes les marques ${ }^{5}$. L'étanchéité, le remontage automatique, la résistance aux chocs, l'usage du quartz sont particulièrement vantés par les constructeurs (Fig. IV et V, cahier couleur). La quatrième section attire l'attention sur l'importance prise par les sujets en lien avec les sports qui sont synonymes de modernité (sport automobile, tennis, ski, patinage, golf, équitation... $)^{6}$. Le sportif

2. Le projet a été dirigé par Régis Huguenin et Nathalie Marielloni, respectivement conservateur et conservatrice-adjointe du Musée international d'horlogerie et par Gianenrico Bernasconi, professeur, directeur de recherche et Catherine Herr-Laporte, assistante-doctorante, à l'Institut d'histoire de l'Université de Neuchâtel. La base de données réalisée peut être consultée par les chercheurs au sein du MIH.

3. Le cheminement de l'exposition, fondé principalement sur l'étude iconographique des affiches et des annonces en relation avec l'histoire de la montre, est l'objet d'une synthèse faite par Régis Huguenin dans l'ouvrage, réunissant une quinzaine d'auteurs, qu'il a dirigé avec Gianenrico Bernasconi, présentant les résultats de la recherche initiée en 2015 dans les deux institutions neuchâteloises : Huguenin, 2019, p. 73-101.

4. Herr-Laporte, 2019, p. 179-197. Nous remarquons ainsi une affiche, datée 1898, d'Hugo d'Alési pour la fabrique Haldy (Besançon) qui met en scène le vieillard et sa faux désignant une nuée de montres tombant sur un paysage rougi par le soleil, déclarant «Elles règlent le soleil » (repr. p. 181). 5. Sur une affiche de la maison Solvil Paul Ditisheim, nous pouvons lire le slogan : «Sa qualité fait sa réclame ".

6. Gogniat, 2019, p. 235-248. 
ou la sportive se doivent d'avoir une montre précise et résistante, si possible un chronographe. La cinquième section souligne la valorisation de la montre par les publicitaires comme un signe de l'élégance et du bon goût ${ }^{7}$. Les femmes et les hommes sont invités à posséder plusieurs montres selon le moment de la journée et leurs activités. Un vêtement, un accessoire, un élément de décor intérieur, une attitude suffisent à évoquer le statut social auquel s'accorde la montre présentée. Une dernière partie regroupe des affiches caractérisées non par le produit fini mais par une image magnifiée du lieu de sa fabrication, la manufacture (Fig. VI, cahier couleur). La puissance de l'entreprise ainsi montrée suffit à donner confiance dans la marque ${ }^{8}$. L'exposition apparaît comme un gigantesque livre qui mettrait en images l'histoire sociale, technique et industrielle de la montre du $\mathrm{Xx}^{\mathrm{e}}$ siècle, tant les affiches en sont le reflet. Notons l'intérêt documentaire des publicités qui renvoient à l'époque glorieuse de maisons disparues comme Doxa, Oris. Jaz.

Les affiches de grand format comme les annonces destinées aux magazines composent un panorama dans lequel il est aisé de reconnaître les styles contemporains successifs, Art nouveau, Art déco, Modernisme. Elles sont empreintes de l'expression personnelle de leur créateur quand la maison d'horlogerie n'impose pas une certaine homogénéité dans ses messages. Des signatures relativement nombreuses peuvent être relevées, témoignant du souci des industriels de solliciter des dessinateurs reconnus dans les autres domaines du luxe. Citons Leonetto Cappiello (Jaeger, Longines), Charles Lemmel (Doxa, Universal), René Bleuer (Longines), Jean d'Ylen (Longines, Vulcain), Herbert Leupin (Tissot), Kiraz (Maurice Lacroix).

L'exposition et la publication, intitulées tel un slogan L'heure pour tous, une montre pour chacun, contribuent de façon inédite à la connaissance de l'histoire sociale de l'horlogerie. Jusqu'à présent, le sujet n'avait jamais été traité avec cette ampleur ni pour lui-même. Il a été abordé dans quelques articles consacrés à une marque ou dans le cadre de monographies ${ }^{9}$. Quand

7. Le mot "élégance " revient sans cesse d'une marque à l'autre. Rappelons le célèbre slogan de Longines, accompagné du portrait d'Audrey Hepburn : Elegance is an attitude.

8. Marielloni, 2019, p. 159-178.

9. Nous pensons à l'article d'Estelle Fallet (Fallet, 2002) ou à des monographies comme celle d'Henri Marquis (Marquis, 1995). Il faut remonter à 1932 pour repérer une publication (issue d'une thèse) : Montandon, 1932. L'ouvrage marquant le cinquantenaire du Journal suisse d'horlogerie, fondé en 1876, intitulé Le livre d'or de l'horlogerie, offre une rétrospective, abondamment 
elles étaient exposées, reconnaissons que les affiches servaient surtout à agrémenter les vitrines en les faisant voisiner avec les montres des fabricants. Avec cette double réalisation, muséographique et scientifique, le champ de la recherche sur la publicité horlogère est enfin brillamment ouvert.

Catherine Cardinal

Université Clermont-Auvergne

\section{Bibliographie}

Fallet Estelle, "Tissot s'affiche. Une histoire en images ", Chronométrophilia, $n^{\circ} 53,2002$, p. 11-28.

Gogniat Jérôme, «Le sport et la modernité dans la publicité horlogère dans la première moitié du Xx siècle ", dans Huguenin Régis, Bernasconi Gianenrico (dir.), L'heure pour tous, une montre pour chacun. Un siècle de publicité horlogère, Éditions Alphil, Neuchâtel, 2019, p. 235-248.

Herr-Laporte Catherine, "Le temps et sa représentation dans les publicités horlogères du $\mathrm{xx}^{\mathrm{e}}$ siècle ", dans Huguenin Régis, Bernasconi Gianenrico (dir.), L'heure pour tous, une montre pour chacun. Un siècle de publicité horlogère, Éditions Alphil, Neuchâtel, 2019, p. 179-197.

Huguenin Régis, Bernasconi Gianenrico (dir.), L'heure pour tous, une montre pour chacun. Un siècle de publicité horlogère, Éditions Alphil, Neuchâtel, 2019.

Huguenin Régis, "L'heure pour tous, une montre pour chacun. Un siècle de publicité horlogère (1880-1980) ", dans Huguenin Régis, Bernasconi Gianenrico (dir.), L'heure pour tous, une montre pour chacun. Un siècle de publicité horlogère, Éditions Alphil, Neuchâtel, 2019, p. 73-101.

Le livre d'or de l'horlogerie, Journal suisse d'horlogerie, Genève, Neuchâtel, 1926.

Marielloni Nathalie, "L'image de l'usine et de ses moyens de production ", dans Huguenin Régis, Bernasconi Gianenrico (dir.), L'heure pour tous, une montre pour chacun. Un siècle de publicité horlogère, Éditions Alphil, Neuchâtel, 2019, p. 159-178.

Marquis Henri, Comment habiller le temps, un siècle de design horloger Juvenia, Scriptar, Lausanne, 1995.

Montandon Carlo, La publicité dans l'industrie horlogère suisse, Bovard-Gidey, Lausanne, 1932.

illustrée par les publicités des maisons horlogères, qui témoigne de l'intérêt du sujet longtemps négligé. 